\title{
Isolation and characterization of endophytic fungi having plant growth promotion traits that biosynthesizes bacosides and withanolides under in vitro conditions
}

\author{
Sumit K. Soni ${ }^{1} \cdot$ Rakshapal Singh $^{2} \cdot$ Nem K. Ngpoore $^{3} \cdot$ Abhishek Niranjan $^{3} \cdot$ Purnima Singh $^{1} \cdot$ Aradhana Mishra $^{1}$. \\ Sudeep Tiwari ${ }^{4}$ (i)
}

Received: 16 March 2021 / Accepted: 18 July 2021 / Published online: 2 August 2021

(c) Sociedade Brasileira de Microbiologia 2021

\begin{abstract}
Endophytes are regarded with immense potentials in terms of plant growth promoting (PGP) elicitors and mimicking secondary metabolites of medicinal importance. Here in the present study, we explored Bacopa monnieri plants to isolate, identify fungal endophytes with PGP elicitation potentials, and investigate secretion of secondary metabolites such as bacoside and withanolide content under in vitro conditions. Three fungal endophytes isolated (out of 40 saponin producing isolates) from leaves of B. monnieri were examined for in vitro biosynthesis of bacosides. On morphological, biochemical, and molecular identification (ITS gene sequencing), the isolated strains SUBL33, SUBL51, and SUBL206 were identified as Nigrospora oryzae (MH071153), Alternaria alternata (MH071155), and Aspergillus terreus (MH071154) respectively. Among these strains, SUBL33 produced highest quantity of Bacoside $\mathrm{A}_{3}\left(4093 \mu \mathrm{g} \mathrm{mL^{-1 }}\right)$, Jujubogenin isomer of Bacopasaponin C $\left(65,339 \mu \mathrm{g} \mathrm{mL}^{-1}\right)$, and Bacopasaponin C $\left(1325 \mu \mathrm{g} \mathrm{mL}^{-1}\right)$ while Bacopaside II $\left(13,030 \mu \mathrm{g} \mathrm{mL}^{-1}\right)$ was produced by SUBL51 maximally. Moreover, these aforementioned strains also produced detectable concentration of withanolides-Withaferrin A, Withanolide A $\left(480 \mu \mathrm{g} \mathrm{mL}^{-1}\right)$, and Withanolide B $\left(1024 \mu \mathrm{gL}^{-1}\right)$ respectively. However, Withanolide A was not detected in the secondary metabolites of strain SUBL51. To best of our knowledge, the present study is first reports of Nigrospora oryzae as an endophyte in B. monnieri with potentials of biosynthesis of economically important phytomolecules under in vitro conditions.
\end{abstract}

Keywords Endophytes $\cdot$ Bacopa monnieri $\cdot$ Bacosides $\cdot$ Medicinal plants $\cdot$ Withanolides $\cdot$ Saponin $\cdot$ In vitro biosynthesis

\section{Introduction}

Sumit K. Soni and Sudeep Tiwari contributed equally to this paper

Responsible Editor: Melissa Fontes Landell

Aradhana Mishra

mishramyco@yahoo.com

Sudeep Tiwari

sudeep@post.bgu.ac.il

1 Department of Plant-Microbe Interaction, CSIR-National Botanical Research Institute, Lucknow 226001, India

2 Biological Central Facility, CSIR-Central Institute of Medicinal and Aromatic Plants, Lucknow 226015, India

3 Chemical Instrumentation Facility, CSIR-National Botanical Research Institute, Lucknow 226015, India

4 Department of Geography and Environmental Development, Ben Gurion University of the Negev, P.O.B. 653, Beer-Sheva, Israel
The medicinal plants and its high economical value secondary metabolites are widely used as raw materials for pharmaceutical, cosmetic, and perfumery industries [1]. Globally, a large population (80\%) still relay on herbal products, supplements for primary healthcare, and immune boosting [2]. Therefore, there is a continuous surge in the demands of herbs and herbal products. In the time of COVID-19 pandemic, demands and usage of herbal supplements and drugs are ever-increasing. In Indian subcontinent, in the present scenario, attention towards medicinal plants in day to day life is highly recommended for maintenance of immune system and immune boosting.

Bacopa monnieri, generally known as Brahmi, is widely used in ayurvedic preparations (Indian system of traditional medicine) for treating various ailments such as epilepsy, anxiety, poor memory, neurosis, psychosis, and renaissance 
of sensory organs [3-6]. Moreover, in modern days, it has also been used in remedies of many other diseases including stress, depressant, ulcer, and hepatic infection $[7,8]$. The high economical value and global demands of bacosides consequently boosted the unorganized collections and over exploitation B. monnieri and subsequently leading to sharp reduction of germplasm and causing a massive loss to its natural habitats [9]. Furthermore, bacosides are present in very low quantity in the plant and the extraction procedure requires huge biomass leading to environmental imbalance and accounting this plant as an endangered species. The overexploitations of $B$. monnieri lead them to enter to highly endangered list of medicinal plants in India [9].

Similarly, Withania somnifera (Ashwagandha) is regarded as Indian ginseng with potential therapeutic values [10] for improving body strength and immune systems, anti-aging, hepatic and cardiac cells protection, control cholesterol level, antipyretic, antiulcer, hemopoietic, etc. [11, 12]. The therapeutic potentials of $W$. somnifera are due to the presence of terpenoids saponins collectively known as withanolides which include Withanolide A, Withanolide B, and Withaferrin A. However like B. monnieri, over exploitation of Withania somnifera is also undergoing rapid depletion in its germplasm. Moreover, it is evident that global warming and climate change have impacted on humans and agriculture. The increasing population and food security are a big challenge too; however with depleting land under cultivation area and other challenges form abiotic [13] and biotic stresses, it is hard to maintain yield attributes [1].

In recent years, endophytes are regarded as major sources for potential metabolites such as alkaloids, benzopyranones, benzoquinones, flavonoids, phenols, steroids, terpenoids, tetralones, and xanthones, [14] with array of novel therapeutic values [15]. Endophytic fungi colonize intercellularly or intracellularly within healthy plant tissues [16] and consequently maintain a harmonious symbiotic relationship without causing any apparent harm or disease symptoms within all examined plants $[17,18]$. The endophytes dwelling inside the medicinal plants forms a positive correlation over time and yields secondary metabolites in the same lines as of the host plants [15]. The endophytes isolated from medicinal plants are proved to be involved in modulation of secondary metabolites and production of pharmacologically important substances facilitates nutrient exchange and enzyme activity, enhanced stress resistance in plants, degradation of pollutants, and help in plant growth by producing plant hormones $[12,19]$.

Therefore to cope from aforementioned issues and meet the desired demands, there is urgent need to search an efficient, eco-friendly, cost-effective alternative production of high contents of bacoside and withanolide. In this regard, native endophytic fungi, a simplest eukaryotic microorganism, could be new sources of aforesaid saponins and will protect naturally inhabiting $B$. monnieri and $W$. somnifera resources. The potential of endophytes to produce pharmacologically important secondary metabolites encouraged us to undertake the studies for unexplored native endophytes from B. monnieri and look for potentially important secondary metabolite biosynthesis under in vitro conditions. We hypothesize that the isolated fungal endophytes will mimic secondary metabolites of $B$. monnieri and will scale up yield of Bacosides when compared to in planta.

\section{Material and methods}

\section{Collection of plant material}

The brahmi plants were cultivated in the research fields of CSIR-Central Institute of Medicinal and Aromatic Plants (CSIR-CIMAP, Lucknow, India, an institute of national importance dedicated to medicinal plants research) located at an elevation of $131 \mathrm{~m}$ elevation from sea level $\left(26^{\circ} 16, \mathrm{~N}\right.$, $80^{\circ} 46$, E and the region has semiarid sub-tropical climatic conditions with an average rainfall of $1000 \mathrm{~mm}$ (https://en. climate-data.org/asia/india/uttar-pradesh/Lucknow). A survey to the fields were done and healthy looking plants were collected and transferred to laboratory for further isolation process as described previously in our research article [16].

\section{Isolation of endophytic fungi}

The isolation of endophytic fungi from brahmi plant leaves were carried out as per previous described methods [16, 20]. The leaves were well rinsed with normal tap water for few minutes to remove surface adherent followed by washing with double distilled and surface sterilized with $70 \%$ ethanol solution for $10 \mathrm{~s}$ followed by treatment with $4 \%$ sodium hypochlorite solution for $5 \mathrm{~min}$. Further the leaves were rinsed with sterile double distilled water for $1 \mathrm{~min}(3$ times). The sterilized plant leaves were dried on pre-sterile filer paper and chopped into small pieces ( 3 to $5 \mathrm{~mm}$ ), and transferred on potato dextrose agar (PDA) plates supplemented with streptomycin $\left(1 \mathrm{gm} \mathrm{L}^{-1}\right)$ followed with incubation in BOD incubator at $28 \pm 1{ }^{\circ} \mathrm{C}$ under dark conditions until the growth of fungal hyphae. Afterwards, the hyphae were transferred carefully into fresh PDA plates to get pure cultures $[15,16]$.

\section{Fungal culturing and preparation of fungal crude extract}

The isolated fungal strains were transferred to potato dextrose broth (PDB), incubated at $28 \pm 2{ }^{\circ} \mathrm{C}$ in dark (200 RPM) under constant shaking conditions for 16 days. After incubation, the fungal crude extract was separated from mycelia 
by filtering through cheesecloth. Filtered supernatant was extracted (ratio of 1:1) with ethyl acetate as organic solvent; further, the supernatant was subsequently left for $48 \mathrm{~h}$ at room temperature to properly solubilize the fungal metabolites in the solvent. Afterwards, ethyl acetate fractions were collected through separating funnel and concentrated in vacuo (Bucchi, Rotavapor, India). The concentrated fungal metabolites were further dissolved in $10 \mathrm{~mL}$ of methyl alcohol and subsequently filtered through $0.2 \mu \mathrm{m}$ filters to obtain the crude extract.

\section{Screening of saponins producing endophytic fungi}

The isolated pure fungal cultures were further screened for their ability of saponins production. To look for the saponins presence in crude extract, $5 \mathrm{~mL}$ aliquots of fungal crude extract (FCE) was mixed with $25 \mathrm{~mL}$ of distill water and heated in a microwave for $2 \mathrm{~min}$, followed by shaking vigorously for 1 min with vortex mixture. Afterwards, the mixtures were allowed to stand for $10 \mathrm{~min}$. The occurrence of stable froth is an indicative of saponins presence. We found 40 endophytic fungi that were capable of producing saponins. These saponin positive isolates were further analyzed for their ability of bacoside and withanolide production.

\section{Quantification of Bacoside A content}

For quantification of Bacoside A content we followed protocols of Murthy et al. [21], $250 \mathrm{ml}$ of FCEs were mixed with same volume of ethyl acetate sequentially extracted 2 times; further left overnight with ethyl acetate and process was repeated next day once again for complete extraction of metabolites. The extracted ethyl acetate fractions were pooled and subsequently concentrated using rotavapor (Bucchi, India). The dry residue of metabolites were collected, and further dissolved in $5 \mathrm{~mL}$ of HPLC grade (Sigma Aldrich) methanol and eluted using micropipette. Afterwards, the eluted metabolites were centrifuged at $6000 \mathrm{rpm}$ for 5 min (Sigma Aldrich) and subsequently supernatant was filtered using $0.45 \mu \mathrm{m}$ nylon syringe filter. The filtrate thus obtained was used for HPLC analysis. The analysis was performed using Shimadzu HPLC (Prominence-model Singapore) operational with LC-20AD pump, SIL-20 AC HT auto-sampler, SPD M20 PDA detector, CTO-10 AS VP column and DGU-14A DEGASSER mobile phase solvent. The reverse phase $\mathrm{C} 18$ column $(250 \mathrm{~mm} \times 0.46 \mathrm{~mm} \times 0.25 \mu \mathrm{m})$ was used. For the mobile phase acetonitrile-water was (with $0.05 \%$ orthophophoric acid) used with gradient solvent system having a run time of $40 \mathrm{~min}$ (from 0-25 $\mathrm{min} 30: 70 \mathrm{v} / \mathrm{v}$, 25-35 $\mathrm{min} 60: 40 \mathrm{v} / \mathrm{v}, 35-37 \mathrm{~min} 60: 40 \mathrm{v} / \mathrm{v}$, and 37-40 $\mathrm{min}$ $30: 70 \mathrm{v} / \mathrm{v}$ ) with the current rate of $1.5 \mathrm{~mL} \mathrm{~min}^{-1}$ [21] The detection was made at $205 \mathrm{~nm}$. The acquisition and computation of data was carried out using lab solution software.
The standard of Bacoside A used was purchased from Natural Remedies Pvt. Ltd., India. A total six fungal isolates were found to synthesize Bacoside A (mixture of four Bacoside standards-Bacoside $\mathrm{A}_{3}$, Bacopaside II, Jujubogenin isomer of Bacopasaponin $\mathrm{C}$, and Bacopasaponin $\mathrm{C}$ ). These fungal isolates were also examined for their withanolide production potentials.

\section{Quantification of Withanolide A, Withanolide B, and Withaferrin A content}

The preparation of fungal metabolite solution for quantification of withanolide content was carried out in the similar lines of the methods described by Chaurasiya et al. [22]. Here also the reverse phase column $(250 \mathrm{~mm} \times 0.46 \mathrm{~mm} \times 0.25 \mu \mathrm{m})$ was used. The mobile phase was water (with $0.1 \%$ acetic acid)-methanol (with $0.01 \%$ acetic acid) with gradient solvent system having run time of $75 \min$ (from 0-30 $\min 60: 40 \mathrm{v} / \mathrm{v}, 30-45 \min 40: 60 \mathrm{v} / \mathrm{v}$, $45-54 \min 25: 75 \mathrm{v} / \mathrm{v}, 54-60 \mathrm{~min} 5: 95 \mathrm{v} / \mathrm{v}$, and 60-75 min $60: 40 \mathrm{v} / \mathrm{v}$ ) with the flow rate of $0.6 \mathrm{~mL} \mathrm{~min}^{-1}$. The detection was made at $227 \mathrm{~nm}$ [22]. The standards of Withanolide A, Withanolide B, and Withaferrin A were procured from Natural Remedies Pvt. Ltd., India.

Out of the six, three best strains producing both bacoside and withanolide were selected for further characterization (biochemical-phytochemical synthesis, extracellular enzymes production, plant growth promoting activities; morphological and molecular-5.8S ITS sequencing and BLAST analysis) and other important studies.

\section{Qualitative screening of other phytochemicals}

The qualitative screening of phytomolecules from fungal crude extracts (FCEs) was performed in the same lines as described by Bandoni et al. [23]. One $\mathrm{mL}$ of FCE was mixed in $1 \mathrm{~mL}$ of chloroform followed by addition of $0.75 \mathrm{~mL}$ of concentrated sulfuric acid. The appearance of reddish-brown precipitate in the interface indicates the presence of terpenoids. For detection of presence of phenols $1 \mathrm{~mL}$ of FCE was transferred to test tube and left for air drying. The air dried crude extract was mixed with $1 \mathrm{~mL}$ of distilled water and a few drops of $\mathrm{FeCl}_{3}$. The appearance of dark green color shows the presence of phenols. To detect the tannins in FCE, $1 \mathrm{~mL}$ of crude extract was mixed with $0.1 \% \mathrm{FeCl}_{3}$. The development of brownish green or a blue black coloration shows the presence of tannins in crude extract. Steroids presence in crude extract were detected by mixing of $1 \mathrm{~mL}$ of FCE with $2 \mathrm{~mL}$ of chloroform and the same volume of concentrated sulfuric acid was added slowly with the mixture. The turning of upper layer into red while green fluorescence by sulfuric acid layer indicates the occurrence of steroids. The alkaloids were looked in by taking $1 \mathrm{~mL}$ of FCE and 
further mixed with $1 \mathrm{~mL}$ of $1 \% \mathrm{HCl}$ solutions in stream bath. Afterwards, few drops of Mayer's reagent were added to the mixture and development of creamish/buff color precipitate indicates the occurrence of alkaloids. The presence of flavonoids was detected by mixing $1 \mathrm{~mL}$ of methanolic FCE with few drops of $1 \%$ ammonia solution. The development of yellow color indicates the occurrence of flavonoids. Anthraquinones were detected in crude extracts by mixing $1 \mathrm{~mL}$ of the FCE with $0.5 \mathrm{~mL}$ of diluted ammonia and shaken. The development of red color indicates the occurrence of anthraquinones. At last to detect glycosides, $1 \mathrm{~mL}$ of glacial acetic acid was added in with $1 \mathrm{~mL}$ of FCE followed by a drop of 5\% ethanolic ferric chloride solution. Further, $1 \mathrm{~mL}$ of concentrated sulfuric acid was carefully dropped down along the sides of test tube. The development of brownish ring between two layers indicated the occurrence of cardiac glycosides [23].

\section{Qualitative screening of extracellular enzymes}

The qualitative screening of extracellular enzymes activities was performed by following the methods described by Sunitha et al. [24]. The 10-day-old fungal cultures (grown at $28 \pm 2{ }^{\circ} \mathrm{C}$ in dark) were used for this purpose. The amylase activity of endophytic fungi was evaluated by inoculating fungal hyphae on starch agar medium (Himedia, India). After 4 days of incubation at $28 \pm 2{ }^{\circ} \mathrm{C}$ in dark, $1 \%$ of iodine solution was poured in culture plates. The appearance of colorless halo zone around fungal colony indicates the positive result of amylase activity. The cellulase activity was examined on PDA (Himedia, India) supplemented with $1 \%$ (w/w) carboxy methyl cellulose (CMC) [25]. After 3 days of incubation at $28 \pm 2{ }^{\circ} \mathrm{C}$ in dark, the culture plates were stained with $2 \%$ of Congo red solution for $5 \mathrm{~min}$ followed by de-staining by washing them with $1 \mathrm{M}$ Sodium chloride solution. The presence of clear halo zone around the colony indicates the positive cellulase activity. For estimating protease and lipase activity, fungal hyphae were inoculated on glycerol casein agar (GCA) and tributyrin (TB) agar medium (Himedia, India) at $28 \pm 2{ }^{\circ} \mathrm{C}$ in dark, respectively. After 4 days of incubation at $28 \pm 2{ }^{\circ} \mathrm{C}$ in dark, clear halo zone around the colony showed positive results. Similarly, laccase activity was evaluated by inoculating fungal hyphae on glucose yeast extract peptone agar medium supplemented with $\alpha$-napthol $\left(0.05 \mathrm{~g} \mathrm{~L}^{-1}\right)$ and incubated at $28 \pm 2{ }^{\circ} \mathrm{C}$ in dark for 4 days. The turning of medium from colorless to blue in color indicates positive laccase activity [24].

\section{Screening of plant growth promoting (PGP) activity}

The qualitative screening of PGP activities such as indole production (IAA), phosphate solubilization, siderophore production, catalase, and antimicrobial activity were assessed for the selected three endophytes. The 10-day-old fungal cultures (grown at $28 \pm 2{ }^{\circ} \mathrm{C}$ in dark) were used for this purpose. The production of IAA activity by endophytic fungi was evaluated by transferring fungal hyphae to PDB and incubated for 4 days on rotary shaker at $200 \mathrm{rpm}$ and $28 \pm 2{ }^{\circ} \mathrm{C}$ in dark. At the end of 4th day, $2 \mathrm{~mL}$ supernatant was separated by centrifugation and mixed with $4 \mathrm{~mL}$ of Salkowski reagent. The appearance of stable pink color showed positive IAA production [26]. For phosphate solubilizing and sidrophore production activity a small disc of fungal hyphae from 10-day-old culture obtained through cork borer and transferred on the culture plates containing Pikovskaya's (PVK) agar medium (HiMedia, India) and CAS agar. The plates were incubated at $28 \pm 2{ }^{\circ} \mathrm{C}$ in dark for 7 days. The appearance of clear zone around the growing colony in PVK indicates positive phosphate solubilization activity [27] whereas sidrophore production was examined by observing the development of deep blue to yellow or orange color zone around the colony in CAS agar [28]. The catalytic activity was examined by growing fungal hyphae on potato dextrose agar medium at $28 \pm 2{ }^{\circ} \mathrm{C}$ in dark for 4 days. An appropriate amount of $\mathrm{H}_{2} \mathrm{O}_{2}$ and was added in culture plates. The liberation of oxygen gas in the form of bubbles indicates the positive catalytic activity.

\section{Antibacterial assay}

The antibacterial activities of FCEs were evaluated by agar diffusion method [29]. Both Gram positive (Bacillus sp.) and Gram negative (Pseudomonas aeruginosa) bacterial strains were tested. The isolated fungal strains were cultured at $28 \pm 2{ }^{\circ} \mathrm{C}$ in dark for 16 days on PDB broth. The mycelia free FCE was separated and used for the assay. The aforesaid bacterial cultures were grown overnight using nutrient broth (HiMedia, India). The supernatant was separated from bacterial cells by centrifugation and $100 \mu \mathrm{L}$ of the cell free culture broth were poured and spread on PDA plates. Afterwards, a $6 \mathrm{~mm}$ well were made in each PDA plates and subsequently loaded with $0.2 \mathrm{~mL}$ of FCE. Streptomycin sulfate $(200 \mathrm{mg} /$ well) was taken as reference for this purpose. The activities of FCEs were calculated by observing the growth inhibition (in $\mathrm{mm}$ ).

\section{Antagonistic assay against pathogenic fungi}

The antagonistic effect of the isolated endophytic fungi was evaluated against Fusarium oxysporum using the dualculture technique [30]. Fusarium oxysporum f. sp. lycopersici (ITCC 1322), obtained from ICAR-Indian Agriculture Research Institute, New Delhi, India, was used for this purpose. Ten-day-old pathogenic fungal culture of $F$. oxysporum was transferred on one side of a fresh PDA plate while the test cultures were inoculated on the other side of the plate 
Fig. 1 Morphological (in PDB and PDA) observation of endophytic fungal strains. (A) Nigrospora oryzae strain SUBL33. (B) Alternaria alternata strain SUBL51. (C) Aspergillus terreus strain SUBL206
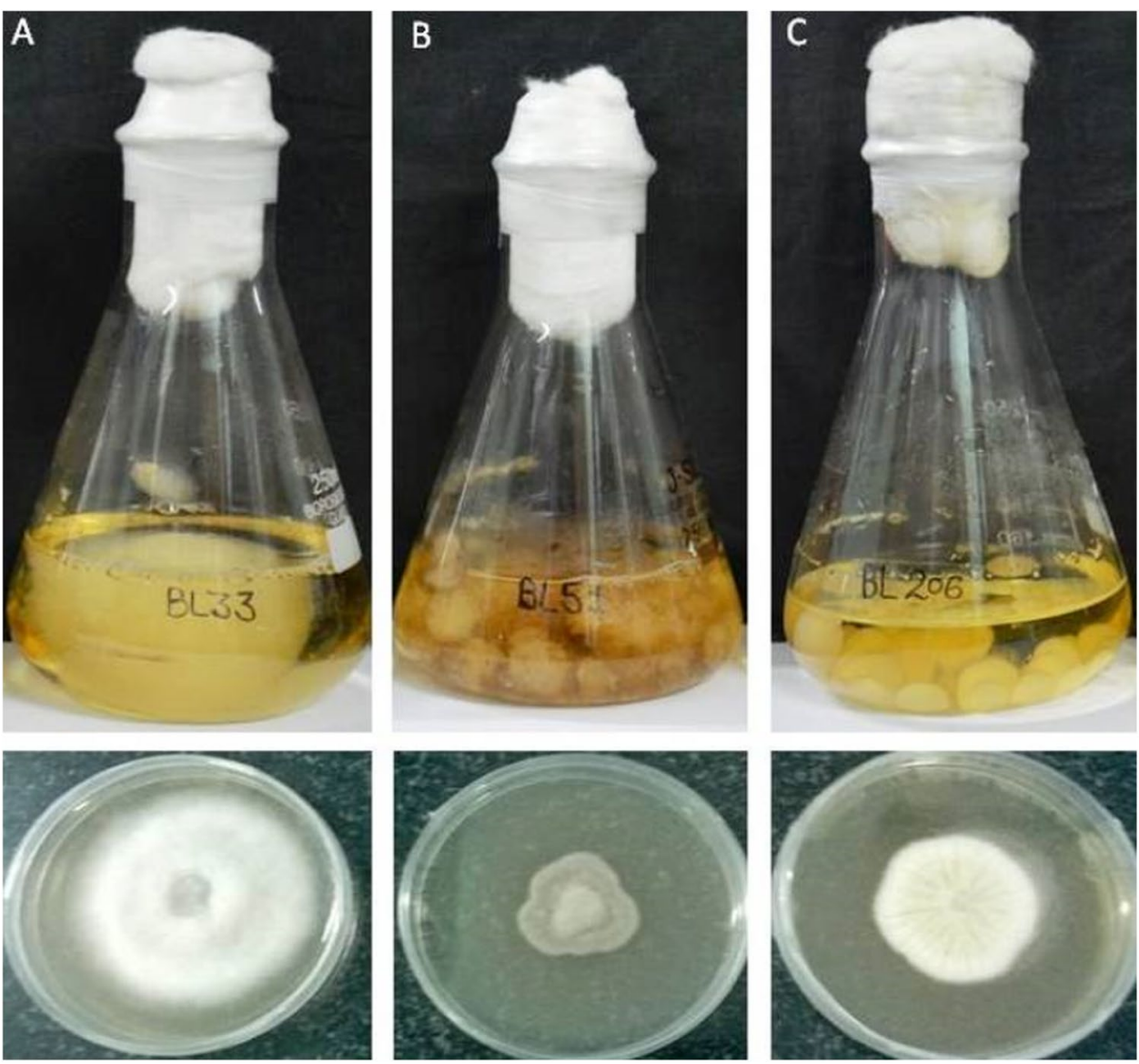

were incubated at $28 \pm 2{ }^{\circ} \mathrm{C}$ for 7 days in dark. While pure culture of $F$. oxysporum inoculated on PDA, plates were used as control. The inhibitions in growth of $F$. oxysporum in presence of test cultures were recorded as positive antagonistic activity.

\section{Morphological and molecular identification of selected endophytic fungi}

The three selected fungal isolates were identified by observing the morphological characteristics on PDA under ambient day light conditions at room temperature. The molecular identification of selected endophytic fungi was performed by amplification and analysis of ITS rDNA sequences. The genomic DNA of endophytic fungi was isolated by following protocols reported by Thakur et al. [31]. Afterwards, the yield and quality of genomic DNA was estimated using Nanodrop spectrophotometer (Nanodrop ND 1000). For amplification of ITS rDNA sequences, the universal primers of Internal Transcribe Spacer 1 (ITS1-5'-TCCGTAGGTGAACCTGCGG-3') and Internal Transcribe Spacer 4 (ITS4-5'-TCCTCCGCTTAT TGATATGC-3') were used. Nearly $25 \mathrm{ng}$ of genomic DNA and 5 pmol of aforementioned primers were used for amplification purpose. The amplifications of ribosomal gene sequence were performed using Mastercyler gradient (Eppendorf) programmed as $95^{\circ} \mathrm{C}$ for $5 \mathrm{~min} ; 32$ cycle at $95^{\circ} \mathrm{C}$ for $30 \mathrm{~s}, 55^{\circ} \mathrm{C}$ for $30 \mathrm{~s}, 72{ }^{\circ} \mathrm{C}$ for $1 \mathrm{~min}$, and $72{ }^{\circ} \mathrm{C}$ for $10 \mathrm{~min}$; and $4{ }^{\circ} \mathrm{C}$ for

Table 1 Blast analysis of isolated endophytic fungal strains

\begin{tabular}{|c|c|c|c|c|c|}
\hline Strains & Identification & GenBank accession no & Similar organism & Accession no & $\begin{array}{l}\text { Sequence } \\
\text { similarity } \\
(\%)\end{array}$ \\
\hline SUBL33 & Nigrospora oryzae & MH071153 & Nigrospora oryzae & KU375674 & 99 \\
\hline SUBL51 & Alternaria alternata & MH071155 & Alternaria alternata & FJ025207 & 99 \\
\hline SUBL206 & Aspergillus terreus & MH071154 & Aspergillus terreus & JX863370 & 98 \\
\hline
\end{tabular}




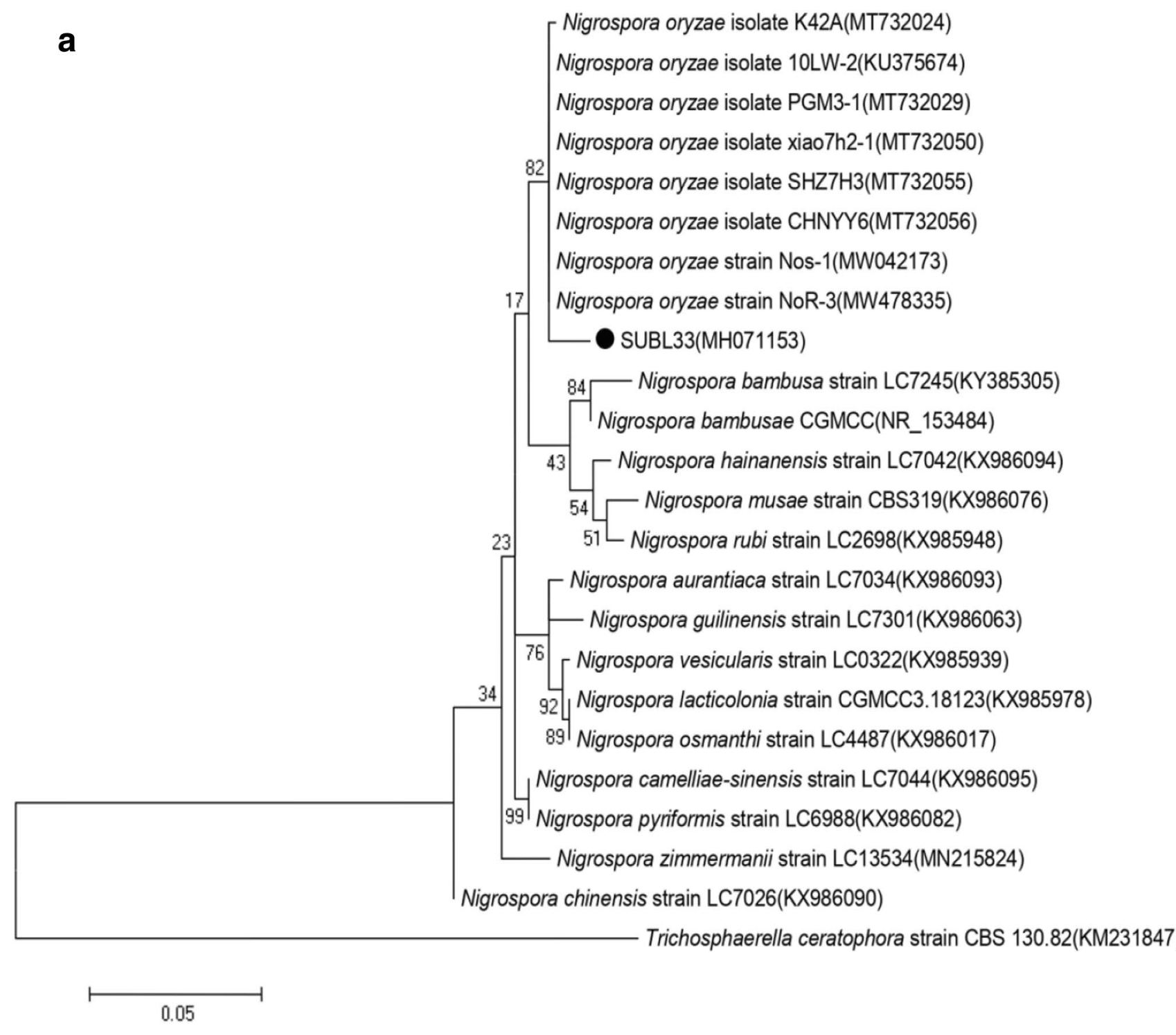

Fig. 2 a Phylogenetic tree constructed from the internal transcribe spacer 1 of $5.8 \mathrm{~S}$ ribosomal RNA of strains SUBL33 and related organisms constructed using maximum likelihood algorithm from an alignment of 534 nucleotides. Accession numbers of corresponding sequences are given in parentheses, and scale bar represents 1 base substitution per 50 nucleotide positions. The bootstrap probabilities calculated from 1000 replications. Trichosphaerella ceratophora strain CBS 130.82 was taken as an out-group. b Phylogenetic tree constructed from the internal transcribe spacer 1 of $5.8 \mathrm{~S}$ ribosomal RNA of strains SUBL51 and related organisms constructed using maximum likelihood algorithm from an alignment of 573 nucleotides. Accession numbers of corresponding sequences are given in

infinite period. The amplified PCR products were purified using PCR Cleanup Kit (Mol Bio, Himedia) by following the instructions mentioned by manufacturers. The PCR product obtained was sequenced by $3130 \mathrm{xl}$ Genetic Analyzer (Applied Biosystems) using sequencing kit (Applied Biosystems, USA) and primer $[11,16]$. The resultant sequence thus obtained was analyzed by nucleotide BLAST (https://blast.ncbi.nlm.nih.gov/). parentheses, and scale bar represents 1 base substitution per 20 nucleotide positions. The bootstrap probabilities calculated from 1000 replications. Setosphaeria rostrata strain MG15 was taken as an outgroup. c Phylogenetic tree constructed from the internal transcribe spacer 1 of 5.8S ribosomal RNA of strains SUBL206 and related organisms constructed using maximum likelihood algorithm from an alignment of 575 nucleotides. Accession numbers of corresponding sequences are given in parentheses, and scale bar represents 1 base substitution per 20 nucleotide positions. The bootstrap probabilities calculated from 1000 replications. Trichocoma paradoxa isolate DWS (19)-23 was taken as an out-group

\section{Phylogenetic analysis and nucleotide sequence accession numbers}

After performing the nucleotide BLAST of sequencing product, the fasta sequence of most similar organisms along with nearest neighbor sequences from the NCBI database were download. Apart from this, one analog sequence of other 


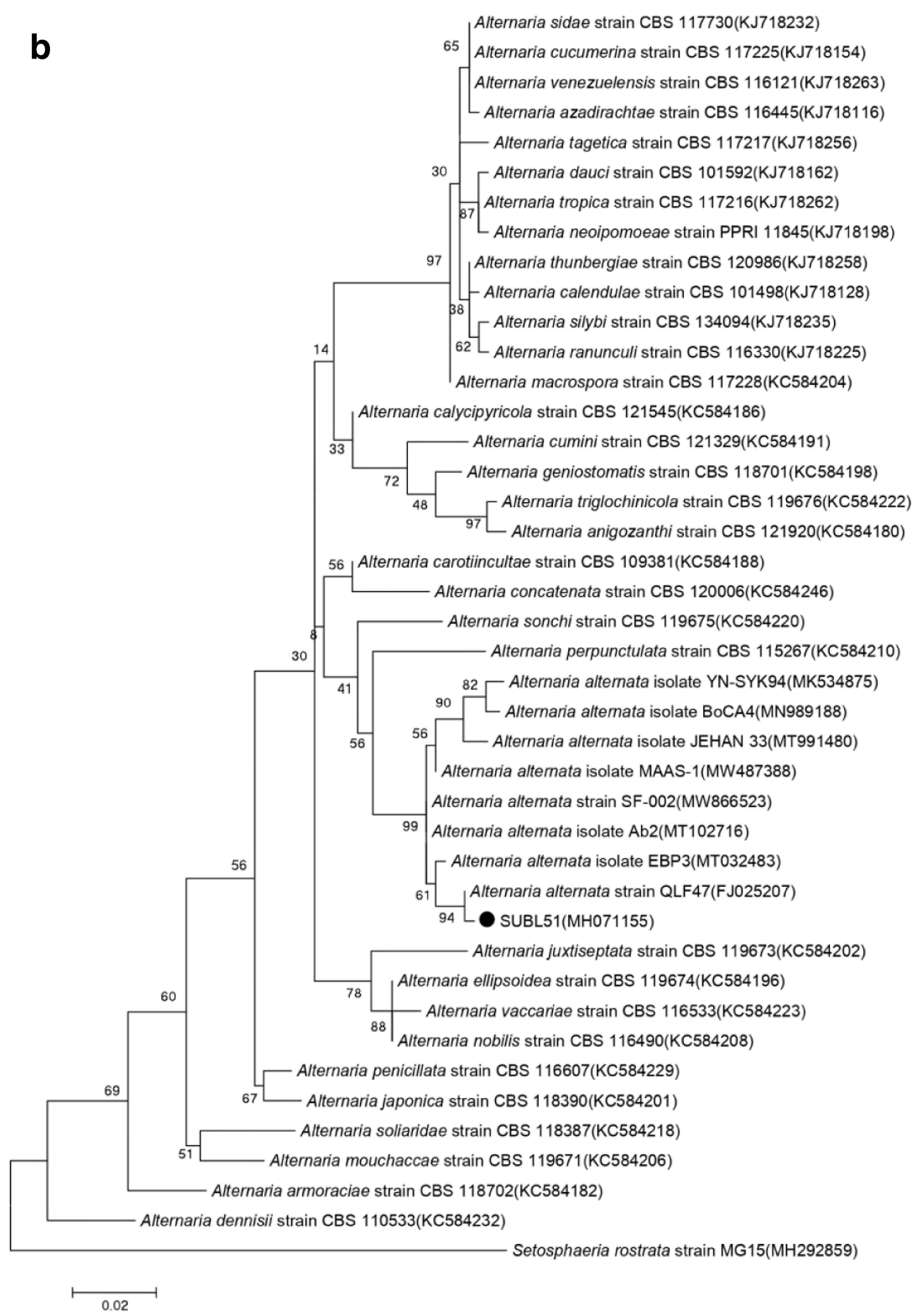

Fig. 2 (continued)

fungal genera was also taken for out group purpose. The downloaded sequences were aligned by inbuilt ClustalW alignment tool of MEGA version 6 software [32]. The construction of phylogenetic tree was carried out by Maximum Likelihood algorithm method using General Time Reversible model with bootstrap replications of 1000 . The ITS rDNA sequences of isolated fungal strain were submitted to GenBank.

\section{Result and discussion}

\section{Screening and identification of endophytic fungal isolates}

The endophytic fungi isolated from leaves of Bacopa monnieri were identified through both morphological (Fig. 1) and molecular characters. It is needed to look on 


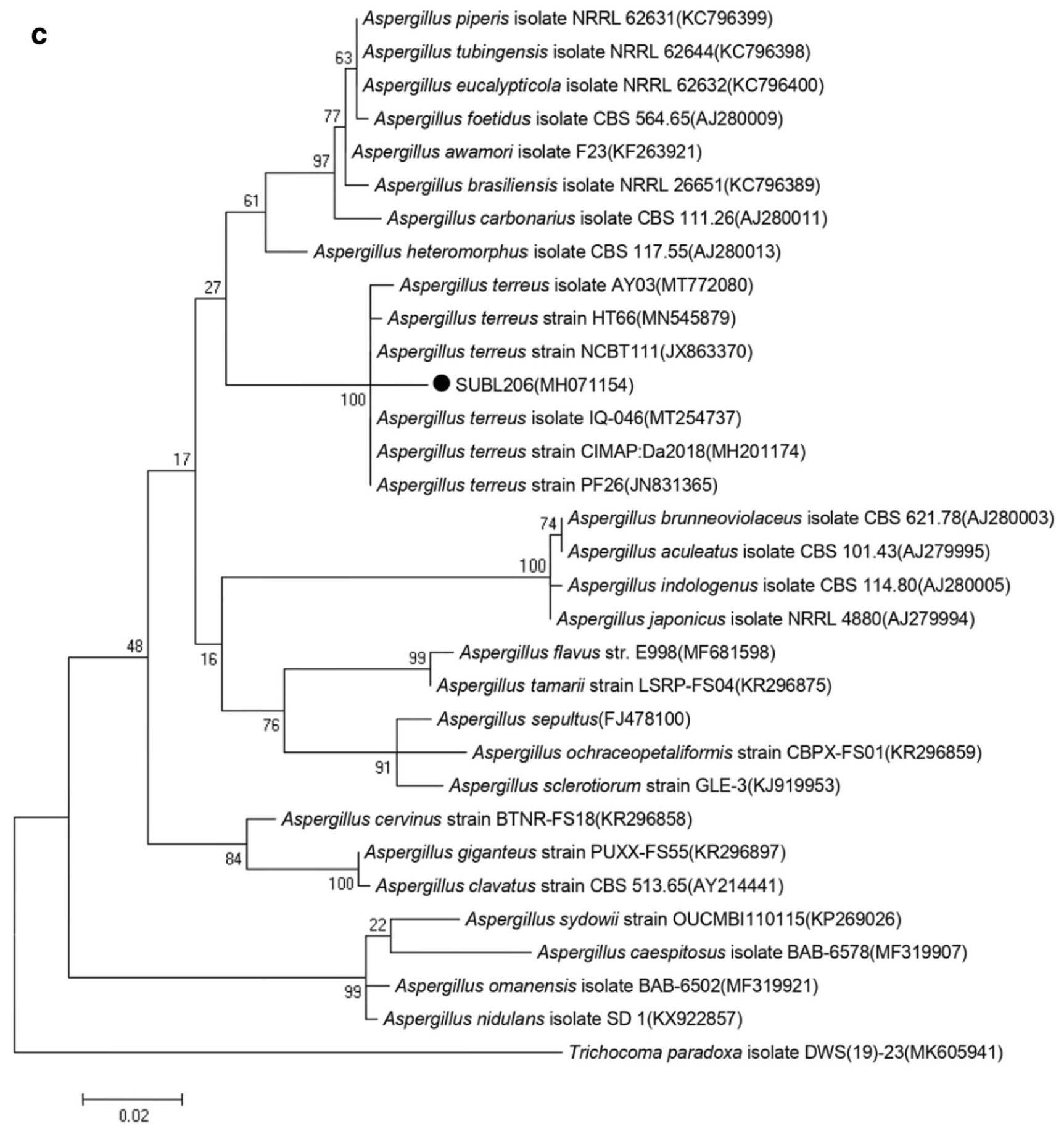

Fig. 2 (continued)

both ways to define and identify the particular endophyte. Tiwari et al. [16] also stresses on using both techniques to reveal the identity of any endophytes. The molecular identification through homology searching (Table 1) and BLAST analysis revealed that the isolated strains namely SUBL33, SUBL51, and SUBL206 belonged to
Nigrospora, Alternaria, and Aspergillus respectively. The aforesaid strains exhibited $99 \%, 99 \%$, and $98 \%$ similarities with Nigrospora oryzae (KU375674), Alternaria alternata (FJ025207), and Aspergillus terreus (JX863370) respectively. The phylogenetic positions of Nigrospora oryzae (strain SUBL33), Alternaria alternata (strain SUBL51), 
Table 2 Qualitative analysis of phytomolecules in crude extract of endophytic fungal strain

\begin{tabular}{llll}
\hline Phytochemical test & \multicolumn{2}{l}{ Strains } & \\
\cline { 2 - 4 } & SUBL33 & SUBL51 & SUBL206 \\
\hline Saponin & + & + & + \\
Terpenoids & + & + & + \\
Phenolics & - & - & + \\
Tannins & - & - & + \\
Steroids & - & - & + \\
Alkaloids & - & + & - \\
Flavonoids & - & - & + \\
Anthraquinones & - & - & - \\
Cardiac glycosides & - & - & - \\
\hline
\end{tabular}

and Aspergillus terreus (strain SUBL206) with other related organisms have been depicted in Fig. 2a, 2b, and 2c. Further, $5.8 \mathrm{~S}$ internal transcribe spacer 1 of Nigrospora oryzae (strain SUBL33), Alternaria alternata (strain SUBL51), and Aspergillus terreus (strain SUBL206) has been submitted to the NCBI GenBank with the accession numbers MH071153, MH071155, and MH071154 respectively.

\section{Screening of phytochemical of endophytic fungi}

The results of qualitative analysis of phytochemical of FCEs were summarized in Table 2 and Fig. S1. The occurrence of phytochemical in endophytes showed that they have potentials to be used as alternative for plantless biosynthesis and in production of economically important phytomolecules for medicinal and industrial use [14, 33]. Saponins [34] and terpenoids [35] have multiple therapeutic values and are found usually in medicinal and aromatic plants. It was found that three isolated endophytic fungi were able to produce saponins and terpenoids which are in similar lines to other reports $[34,36]$. The crude extract of Aspergillus terreus (strain SUBL206) showed the presence of phenolics, tannin, flavonoids, and steroids. The occurrence of phenolic compounds in fungal endophytes also has been reported with marvelous potentials such as antioxidant, antitumor, anti-inflammatory, antimicrobial,

Table 3 Qualitative analysis of extracellular enzyme of endophytic fungal strains

\begin{tabular}{|c|c|c|c|c|c|}
\hline \multirow[t]{2}{*}{ Strains } & \multicolumn{5}{|c|}{ Enzyme screening } \\
\hline & Amylase & Cellulase & Lipase & Protease & Laccase \\
\hline SUBL33 & + & +++ & - & - & - \\
\hline SUBL51 & ++ & - & - & - & - \\
\hline SUBL206 & +++ & ++ & - & +++ & +++ \\
\hline
\end{tabular}

Table 4 Plant growth promoting (PGP) features of endophytic fungal strains

\begin{tabular}{llll}
\hline PGP activity & \multicolumn{2}{l}{ Strains } & \\
\cline { 2 - 4 } & SUBL33 & SUBL51 & SUBL206 \\
\hline IAA & + & + & - \\
P-Solubilization & - & - & - \\
Catalase & + & + & + \\
Sidrophore & - & + & - \\
\hline
\end{tabular}

anti-carcinogenic anti-viral activities [15, 37, 38], chelating metals, and reduce lipoxygenase activity [39, 40]. The production of flavonoids and tannins further showed enhanced antioxidant capacity [15, 41]. Such compounds when used in therapeutic or dietary supplement helps in mitigating the free radicals. The steroids are also important secondary metabolites and are routinely used in medicine due to their antimicrobial and other biological activities [42]. Thus, the phenolic compounds obtained from fungal extract may find place in medicinal preparations for therapeutic purpose. The crude extract of Alternaria alternata (strain SUBL51) showed the positive results of Mayer's test which indicates the presence of alkaloids. The presences of different type of alkaloids in FCEs were reported earlier too exhibited different potentials such as antimicrobial, insecticidal, and anticancer activities [43]. The array of metabolites produced by endophytes may be the contributions of different endophytes in particular plant that are in lines of hosts are specific but not general [44]. Similarly, we found diverse secondary metabolites from different endophytes isolated from B. monnieri might be contributing in vivo in plants for diverse potentials, although all the selected strains were failed to give the positive results of anthraquinones and cardiac glycosides.

\section{Screening of extracellular enzyme of pure cultures}

The results of qualitative analysis of extracellular enzyme of pure cultures were depicted in Table 3 and Fig. S2. All endophytic strains showed positive amylase activity. It is well reported that endophytes utilize starch as a main carbon and energy sources by

Table 5 Antibacterial activity of endophytic fungal strains

\begin{tabular}{lll}
\hline Crude extracts & $\begin{array}{l}\text { Growth inhibition of } \\
\text { Bacillus sp. (in mm) }\end{array}$ & $\begin{array}{l}\text { Growth inhibition } \\
\text { of Pseudomonas aer- } \\
\text { uginosa (in mm) }\end{array}$ \\
\hline Streptomycin (control) & 21 & 17 \\
SUBL33 & NIL & NIL \\
SUBL51 & 8 & NIL \\
SUBL206 & NIL & NIL \\
\hline
\end{tabular}


Table 6 Antagonistic activity of endophytic fungal strains
Fungal strains

Growth of control after 7 days (diameter in $\mathrm{mm}$ )

Growth inhibited by respective strain (in $\mathrm{mm})$

Fusarium oxysporum (control)

Fusarium oxysporum + SUBL33

Fusarium oxysporum + SUBL51

Fusarium oxysporum + SUBL206
34

22

22

27
NA

12

12

7 hydrolyzing them with amylase [19]. The results got strengthened from previous findings and we can predict that in vitro large scale culturing of endophytes needs starch as energy source. Laccase and proteases was produced only by Aspergillus terreus (strain SUBL206). Generally, the fungi that possess the ability to produce laccase are found to mitigate toxic phenols from the medium in which they grow [45]. The production of laccase by endophytic fungi is conformity with the result found earlier by Sunitha et al. [24] where among the isolated fungi, few were able to produced laccase. The enzyme has been also regarded useful in a number of areas such as textile dye transformation, waste detoxification, biosensors, and food technology [46]. We say that the endophytes isolated with laccase potentials will be a good source of the mentioned enzyme and possibility can be exploited in future for the same purpose. Proteases have equally commercial significance like laccase and these enzymes are presently used in broad range of domains such as bioremediation, leather manufacture, animal cell culture, insecticidal agents, silk degumming, detergent, cosmetics, food, and pharmaceuticals industries [47, 48]. Our results indicated Aspergillus terreus (strain SUBL206) produced proteases are in same lines as reported for Aspergillus oryzae [49]. The cellulase enzyme is widely used in pulp and paper industries. We have observed that Nigrospora oryzae (strain SUBL33) and Aspergillus terreus (strain SUBL206) were able to hydrolyze cellulose via the production of cellulase in extracellular medium. The production of extracellular cellulase by endophytic fungi has been well reported [24]. The cellulase production by aforementioned fungi indicates that endophytes have own genetic mechanism necessary to generate cellulase, and this might be used by endophytic fungi for establishing itself in host plant.
Fig. 3 Antimicrobial activity. (A) Antibacterial activity in fungal crude extract against Bacillus sp. and Pseudomonas aeruginosa. (B) Antifungal activity of endophytic fungal strain against Fusarium oxysporum
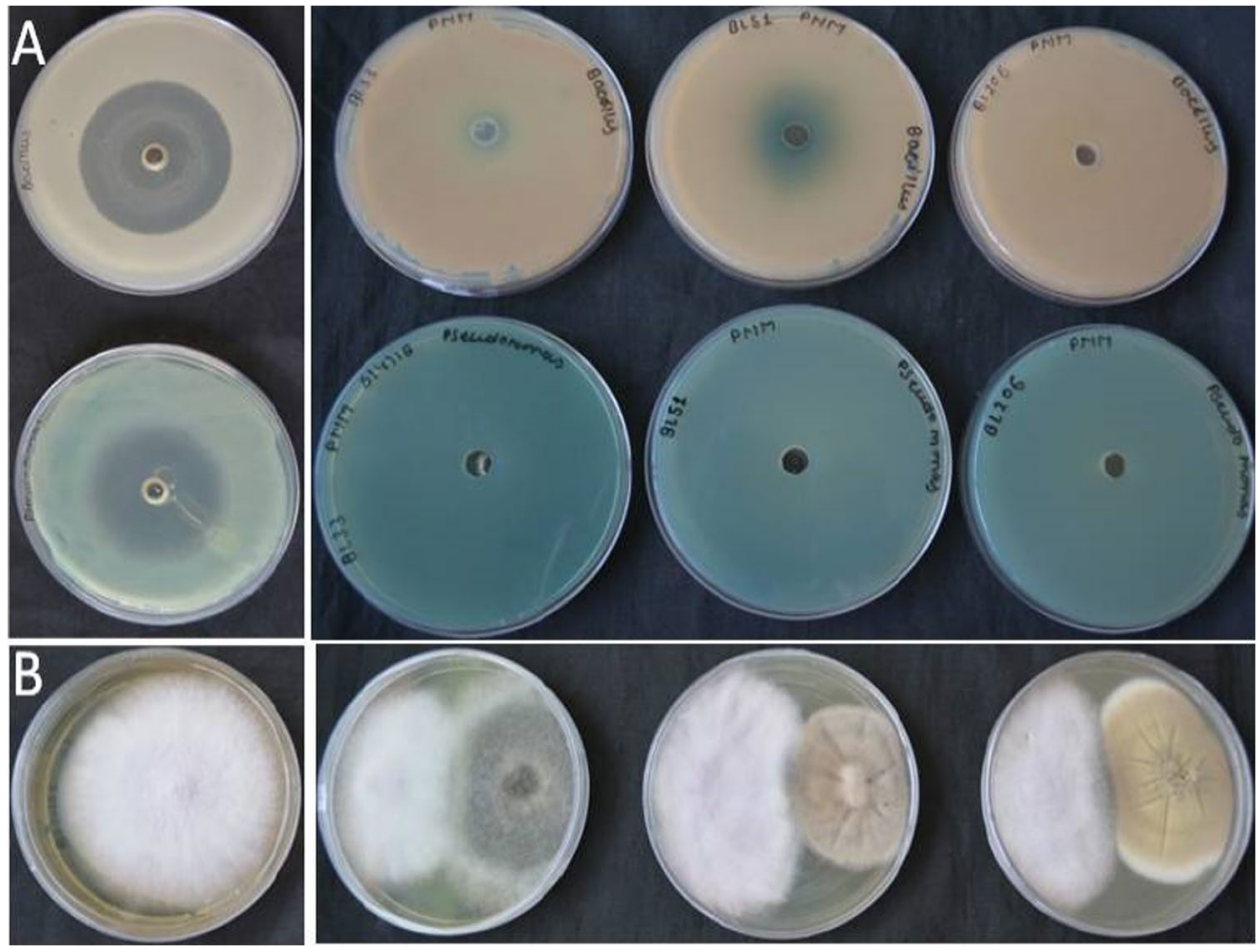

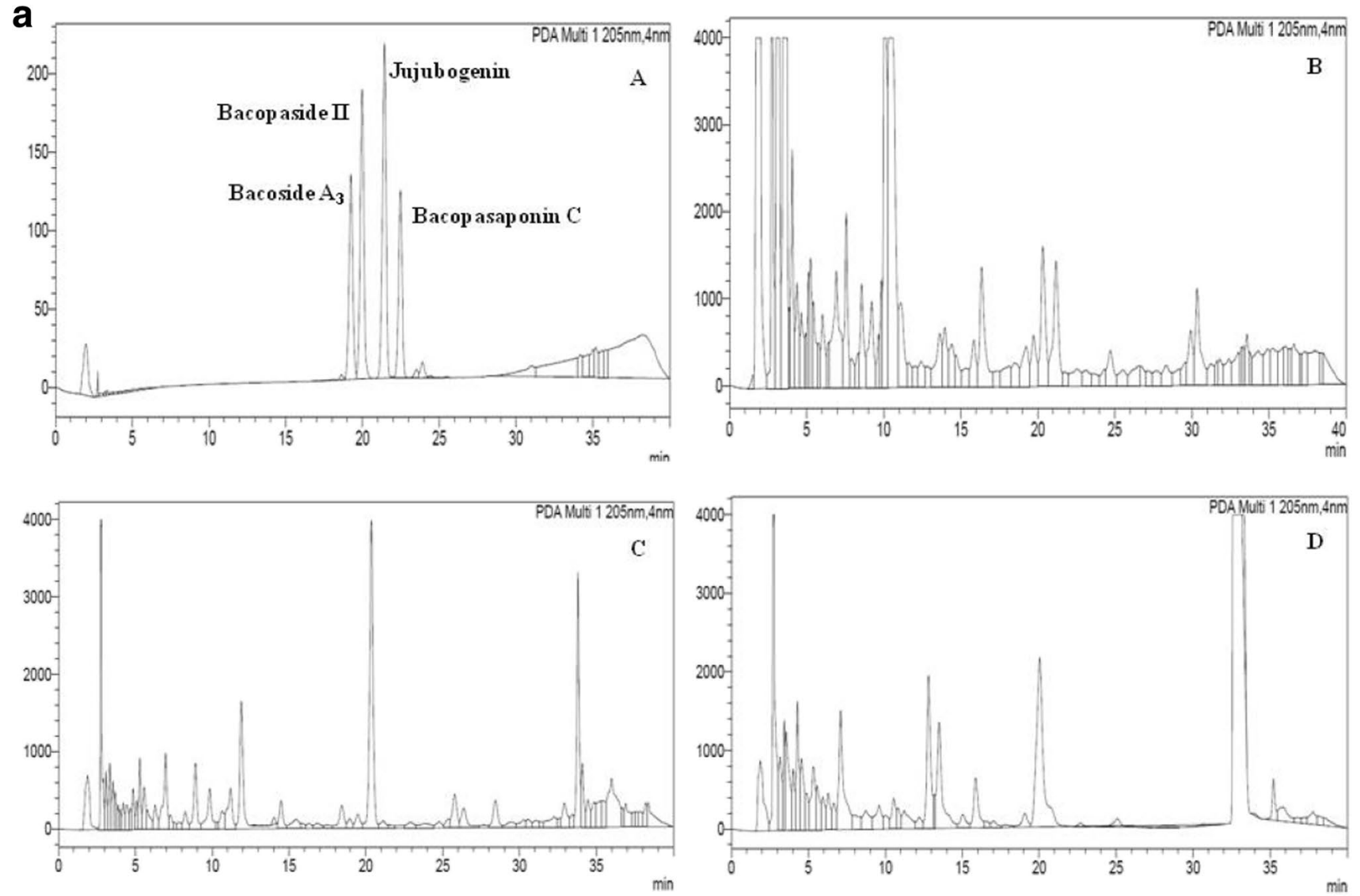

Fig. 4 a HPLC chromatograms of (A) Bacoside A (mixtures of Bacoside $\mathrm{A}_{3}$, Bacopaside II, Jujubogenin isomer of Bacopasaponin $\mathrm{C}$, and Bacopasaponin C). (B) Crude extract of Nigrospora oryzae (strain SUBL33). (C) Crude extract of Alternaria alternata (strain SUBL51). (D) Crude extract of Aspergillus terreus (strain SUBL206). b HPLC

\section{Screening of plant growth promoting (PGP) activity}

The PGP activities of fungal strains were represented in Table 4 and Fig. S3. All strains were found to have catalase activity. Nigrospora oryzae (strain SUBL33) and Alternaria alternata (strain SUBL51) showed the positive IAA test. Moreover, Alternaria alternata (strain SUBL51) also showed sidrophore activity. The production of IAA and sidrophore by endophytic fungi were reported earlier in many studies [50, 51]. However, all aforementioned strains were unable to solubilize phosphate. The PGP activities of endophytes directly attributed to their indole production, phosphorus mobilization, and ammonia production, scavenging free radicals, synthesis of enzymes or metabolites that notably inhibit the growth of pathogenic microorganisms [50] and help plants to remain healthy. Furthermore, the microbes with PGP potentials either endophytic or rhizospheric, supports plant growth and development with secretion of plant growth promoting enzymes [52]. They (microbes) also contribute in enhancement $[1,11,52]$ and

chromatograms of (A) withanolides (mixtures of Withanolide A, Withanolide B and withaferin). (B) Crude extract of Nigrospora oryzae (strain SUBL33). (C) Crude extract of Alternaria alternata (strain SUBL51). (D) Crude extract of Aspergillus terreus (strain SUBL206)

modulation of secondary metabolites in planta [12]. Therefore, microbes with such potentials will be beneficial for targeted enhanced metabolite productions.

\section{Detection of antibacterial and antagonistic activity}

The antibacterial activity of extracellular fungal extract has represented in Table 5 whereas the antagonistic activities with respect to $F$. oxysporum f. sp. lycopersici (ITCC 1322) has depicted in Table 6 . The antibacterial activity of endophytes was examined against both Gram positive (Bacillus sp. GenBank no. JN700911) and Gram negative (Pseudomonas aeruginosa strain CRC5 (GenBank no. HQ995502 and microbial type culture collection no. MTCC 9800)) bacteria. The antibacterial activity of isolated endophytic fungi tested against Gram positive and Gram negative bacteria by well diffusion method. Only the fungal extract of Alternaria alternata (strain SUBL51) showed $8 \mathrm{~mm}$ inhibition zone against Gram positive Bacillus sp.; however, it failed to inhibit the growth of Gram negative. 

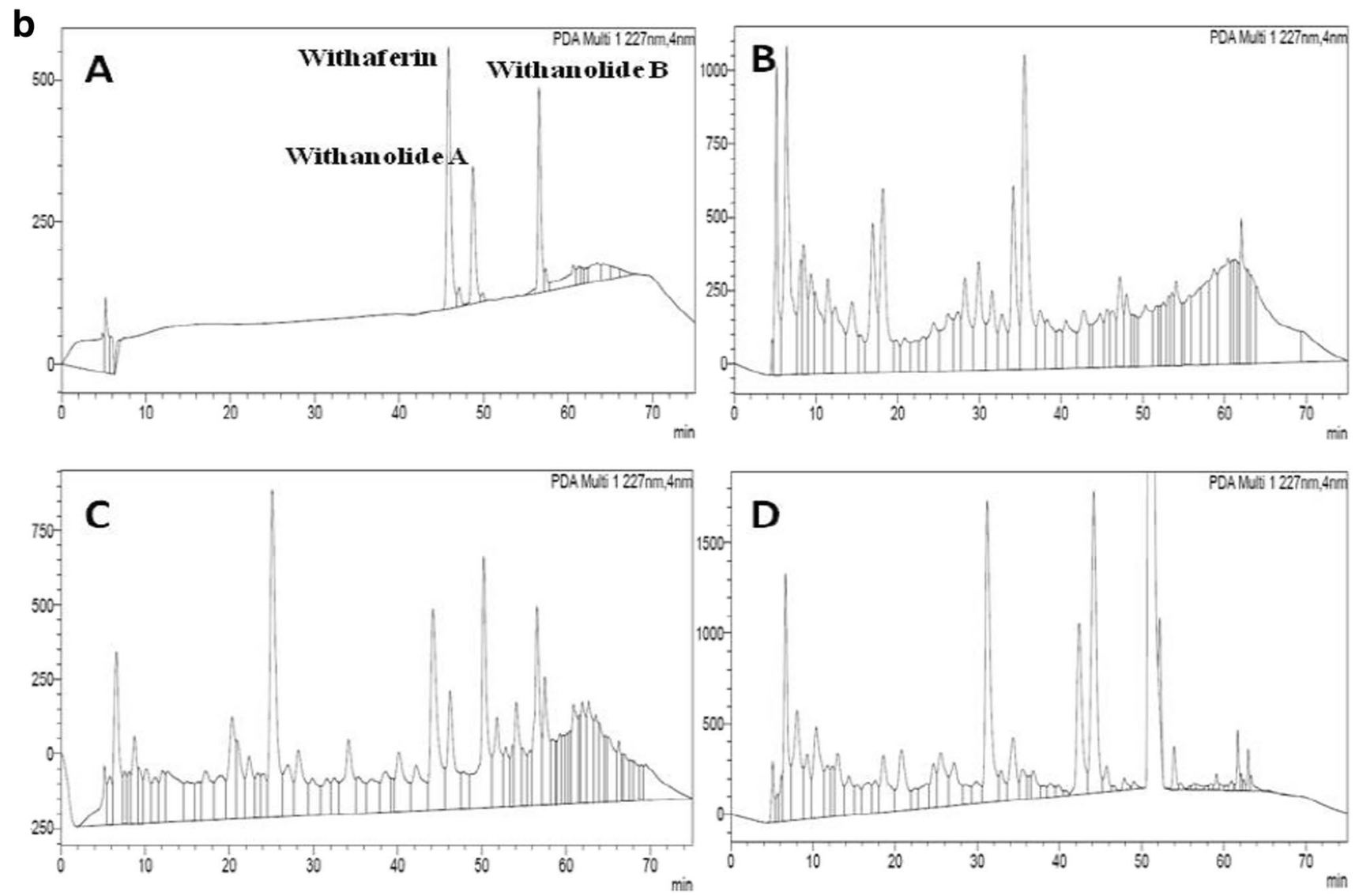

Fig. 4 (continued)

There was no antimicrobial activity observed with Nigrospora oryzae (strain SUBL33) and Aspergillus terreus (strain SUBL206) (Table 5). Our results are as par to findings of other researchers $[53,54]$ where they reported antimicrobial activity of Aspergillus spp. against both Gram positive and Gram negative bacteria. The antagonistic activity of selected endophytes was done against a phytopathogen F. oxysporum f. sp. lycopersici (ITCC 1322) (Fig. 3 and Table 6). The maximum growth was inhibited by Alternaria alternata (strain SUBL51) followed by Nigrospora oryzae (strain SUBL33) and Aspergillus terreus (strain SUBL206) respectively. The antagonistic action of endophytic fungi

Table 7 Analysis of Bacoside A in fungal crude extract

\begin{tabular}{lllll}
\hline Strains & \multicolumn{4}{l}{ Phytomolecules $\left(\mu \mathrm{g} \mathrm{mL}^{-1}\right)$} \\
\cline { 2 - 5 } & Bacoside $\mathrm{A}_{3}$ & Bacopaside II & $\begin{array}{l}\text { Jujubogenin } \\
\text { isomer of Baco- } \\
\text { pasaponin C }\end{array}$ & $\begin{array}{l}\text { Baco- } \\
\text { pasap- } \\
\text { onin C }\end{array}$ \\
\hline SUBL33 & 4093 & 7114 & 65,339 & 1325 \\
SUBL51 & 1191 & 13,030 & 554 & 439 \\
SUBL 206 & 1339 & 12,178 & *ND & 354 \\
\hline
\end{tabular}

*ND, not detectable against phytopathogens tested may be attributed either by production of antibiotics or cell wall degrading enzymes [55]. These potentials might be also responsible for protecting plants from naturally from different fungal diseases and enhanced immunity.

\section{HPLC analyses of Bacoside A content}

The culture filtrates of Nigrospora oryzae (strain SUBL33), Alternaria alternata (strain SUBL 51), and Aspergillus terreus (strain SUBL206) were subjected to HPLC for analysis of bacosides. As a reference, Bacoside A (mixture of four Bacoside standards-Bacoside

Table 8 Analysis of withanolides-Withaferrin A, Withanolide A, and Withanolide $\mathrm{B}$ in fungal crude extract

\begin{tabular}{llll}
\hline Strains & \multicolumn{2}{l}{ Phytomolecules $\left(\mu \mathrm{g} \mathrm{mL}^{-1}\right)$} \\
\cline { 2 - 4 } & Withaferrin A & Withanolide A & Withanolide B \\
\hline SUBL33 & 174 & 168 & 606 \\
SUBL51 & 480 & $* \mathrm{ND}$ & 1024 \\
SUBL206 & 18 & 72 & 48 \\
\hline
\end{tabular}

*ND, not detectable 
$\mathrm{A}_{3}$, Bacopaside II, Bacopasaponin $\mathrm{C}$, and Jujubogenin isomer of Bacopasaponin C) was used. According to the spectra (Fig. 4aa and Table 7), all the endophytes Nigrospora oryzae (strain SUBL33), Alternaria alternata (strain SUBL51), and Aspergillus terreus (strain SUBL206) produced significant concentrations of Bacoside A (Table 7). Among aforesaid strains, SUBL33 produce highest quantity of Bacoside A3 $\left(4093 \mu \mathrm{g} \mathrm{mL}{ }^{-1}\right)$, Jujubogenin isomer of Bacopasaponin $\mathrm{C}\left(65,339 \mu \mathrm{g} \mathrm{mL}^{-1}\right)$, and Bacopasaponin $\mathrm{C}\left(1325 \mu \mathrm{g} \mathrm{mL}^{-1}\right)$ while Bacopasaponin II was produced maximum by SUBL51 (13,030 $\mu \mathrm{g} \mathrm{mL}^{-1}$ ) (Table 7). Although, Jasim et al. [36] have characterized and reported the synthesis of bacoside from Aspergillus spp. under in vitro conditions but it is a novelty of our work which reports both withanolides and bacosides from endophytes including A. terreus in same metabolites in reasonable quantity.

\section{HPLC analyses of Withanolide A, Withanolide B, and Withaferrin A content}

The enhance production of withanolide through modulation of its pathway has been earlier reported by our laboratory [12]. There are also a very few report of production of Withanolide from endophytic fungi [56]. However endophytes from B. monnieri biosynthesizing both the phytomolecules which is obtained from different medicinal plants are unique. The fungal crude extracts were looked in for detection of Withanolide A, Withanolide B, and Withaferrin A content using HPLC (Fig. 4b and Table 8). As a reference, mixtures of aforesaid phytochemical were used as standards. As spectral analysis it was found that Nigrospora oryzae (strain SUBL33), Alternaria alternata (strain SUBL51), and Aspergillus terreus (strain SUBL206) all produced detectable concentrations of withanolide (Fig. $4 \mathrm{~b}$ and Table 8). The endophytes Alternaria alternata (strain SUBL51) produced withaferrin A and Withanolide B phytochemical $\left(480 \mu \mathrm{g} \mathrm{mL}^{-1}\right.$ and $\left.1024 \mu \mathrm{g} \mathrm{mL}^{-1}\right)$ respectively, highest quantity when compared with other two strains (Table 8). However, Alternaria alternata (strain SUBL51) was unable to produce Withanolide A, which was reported highest in Nigrospora oryzae (strain SUBL33). Therefore, we believe that the unique endophytes with dual properties of biosynthesizing both the phytomolecules are encoring and will prospect for future targets of scale-up studies and therapeutic in vivo studies using model systems.

\section{Conclusion}

This is the first report of biosynthesis and production of bacosides and withanolides through endophytes from $B$. monnieri under in vitro conditions. The isolated native endophytic fungi which have PGP potentials could be utilized for plantless, efficient production of bacosides and withanolides in a short period of time with eco-friendly and cost-effective manner. The endophytes if utilized for commercial purpose will minimize unorganized collection and over exploitation of $B$. monnieri and $W$. somnifera and will protect the rapid depletion of their germplasm and ultimately boost their survival in natural habitats. Moreover, this study will strengthen the importance of endophytes mimicking phytomolecules of economic importance. Further, it will encourage the researchers to explore endophytes from different medicinal and aromatic plants for biosynthesis of phytomolecules in demand in pharmaceutical and phytochemical industries, and thus will also help in minimizing the cost and adverse impacts on nature.

Supplementary Information The online version contains supplementary material available at https://doi.org/10.1007/s42770-021-00586-0.

Acknowledgements The authors wish to thank the Director, CSIRNational Botanical Research Institute, Lucknow, India, for providing necessary facilities and encouragement during the course of investigation and the Science and Engineering Research Board-Department of Science and Technology (SERB-DST), India, for providing financial support to SKS in the form of National-Post Doctoral Fellowship.

Author contribution S.K.S and A.M. conceived and designed the experiment. S.K.S performed the work related to microbiology and P.S. helped in performing the experiment, A.N and N.K.N. performed the high-performance liquid chromatography and analyzed the results. S.K.S. performed the sequencing of microbes and RS too helped in experimentation. S.K.S. and S.T analyzed the data and wrote the paper. All authors have read and approved the manuscript.

Funding S.K.S is thankful to Science and Engineering Research Board-Department of Science and Technology (SERB-DST), India, providing financial Support in the form of National-Post Doctoral Fellowship (Grant number PDF/2016/000531) during the entire course of investigation.

This article does not contain any studies with animals or human participants performed by any of the authors.

\section{Declarations}

Conflict of interest The authors declare no competing interests.

\section{References}

1. Tiwari S, Pandey S, Singh PC, Pandey R (2017) Biocontrol agents in co-inoculation manages root knot nematode [Meloidogyne incognita (Kofoid \& White) Chitwood] and enhances essential oil content in Ocimum basilicum L. Ind Crop Prod.https://doi.org/ 10.1016/j.indcrop.2016.12.030

2. Ekor M (2014) The growing use of herbal medicines: issues relating to adverse reactions and challenges in monitoring safety. Front Pharmacol 4:177. https://doi.org/10.3389/fphar.2013.00177 
3. Mathur S, Gupta MM, Ram M, Sharma S, Kumar S (2002) Herb yield and bacoside A content of field grown Bacopa monniera accessions. J Her Spi Med Plan 9:11-18

4. Singh HK, Dhawan BN (1997) Neuropsychopharmacological effects of the Ayurvedic nootropic Bacopa monniera Linn (Brahmi). Ind J Pharmachol 29:5359-5365

5. Phulara S, Shukla V, Tiwari S, Pandey R (2015) Bacopa monnieri promotes longevity in Caenorhabditis elegans under stress conditions. Pharmacogn Mag 11(42):410-416

6. Nemetchek MD, Stierle AA, Stierle DB, Lurie DI (2017) The Ayurvedic plant Bacopa monnieri inhibits inflammatory pathways in the brain. J Ethnopharmacol 197:92-100

7. Sumathy T, Nongbri A (2008) Hepatoprotective effects of Bacoside-A, a major constituent of Bacopa monniera Linn. Phytomedicine 15:801-905

8. Gupta R, Tiwari S, Saikia KS, Shukla V, Singh R, Singh SP, Kumar APV, Pandey R (2014) Exploitation of microbes for enhancing bacoside content and reduction of Meloidogyne incognita infestation in Bacopa monnieri L. Protoplasma.https://doi. org/10.1007/s00709-014-0657-5

9. Prasad R, Kamal S, Sharma PK, Oelmüller R, Varma A (2013) Root endophyte Piriformospora indica DSM 11827 alters plant morphology, enhances biomass and antioxidant activity of medicinal plant Bacopa monniera. J Basic Microbiol 53(12):1016-1024

10. Sharma V, Sharma S, Pracheta PR (2011) Withania somnifera: a rejuvenating Ayurvedic medicinal herb for the treatment of various human ailments. Int J Pharmtech Res 3:187-192

11. Tiwari Sudeep, Saikia Shilpi K, Singh Rashmi, Singh ShiveshPratap, Pandey Rakesh (2014) Native microbial inoculants for the management of Meloidogyne incognita in Withania somnifera $\mathrm{cv}$. Poshita. Proc Natl Acad Sci India Sect B Biol Sci 86(1):55-63. https://doi.org/10.1007/s40011-014-0403-y

12. Pandey SS, Singh S, Pandey H, Srivastava M, Ray T, Soni S, Pandey A, Shanker K, Babu CSV, Banerjee S, Gupta MM, Kalra A (2018) Endophytes of Withania somnifera modulate in planta content and the site of withanolide biosynthesis. Sci Rep 8(1):5450

13. Soni SK, Singh R, Awasthi A et al (2013) A Cr(VI)-reducing Microbacterium sp. strain SUCR140 enhances growth and yield of Zea mays in $\mathrm{Cr}(\mathrm{VI})$ amended soil through reduced chromium toxicity and improves colonization of arbuscular mycorrhizal fungi. Environ Sci Pollut Res 21:1971-1979

14. Tan RX, Zou WX (2001) Endophytes: a rich source of functional metabolites. Nat Prod Rep 18(4):448-459

15. Palanichamy P, Krishnamoorthy G, Kannan S, Marudhamuthu M (2018) Bioactive potential of secondary metabolites derived from medicinal plant endophytes. Egypt J Basic Appl Sci 5(4):303-312

16. Tiwari S, Singh S, Pandey P, Saikia S K, Negi A S, Gupta S K, Pandey R, Banerjee S (2014a) Isolation, structure determination and anti-aging effects of 2,3-pentanediol from endophytic fungus of Curcuma amada and docking studies. Protoplasma. https://doi. org/10.1007/s00709-014-0618-0

17. Chandra S (2012) Endophytic fungi: novel sources of anticancer lead molecules. Appl Microbiol Biotechnol 95:47-59

18. Mishra A, Singh SP, Mahfooz S, Bhattacharya A, Mishra N, Shirke PA, Nautiyal CS (2018) Bacterial endophytes modulates the withanolide biosynthetic pathway and physiological performance in Withania somnifera under biotic stress. Microbiol Res 212:17-28

19. Desire MH, Bernard F, Forsah MR, Assang CT, Denis ON (2014) Enzymes and qualitative phytochemical screening of endophytic fungi isolated from Lantana camara Linn. leaves. $\mathrm{J}$ Appl Biol Biotechnol 2(6):1-6

20. Bhardwaj A, Sharma D, Jadon N, Agrawal PK (2015) Antimicrobial and phytochemical screening of endophytic fungi isolated from spikes of Pinus roxburghii. Arch clin microbiol 6(3):1
21. Murthy PBS, Raju VR, Ramakrisana T, Chakravarthy MS, Kumar KV, Kannababu S, Subbaraju GV (2006) Estimation of twelve bacopa saponins in Bacopa monnieri extracts and formulations by high-performance liquid chromatography. Chem Pharm Bull 54(6):907-911. https://doi.org/10.1248/cpb.54.907

22. Chaurasiya ND, Uniyal CG, Lal P, Misra L, Sangwan NS, Tuli R, Sangwan RS (2008) Analysis of withanolides in root and leaf of Withania somnifera by HPLC with photodiode array and evaporative light scattering detection. Phytochem Anal 19:148-154. https://doi.org/10.1002/pca.1029.23

23. Bandoni AL, Mendiondo ME, Rondina RVD, Coussio JD (1976) Survey of Argentine medicinal plants, folklore and phytochemical screening II. Econ Bot 30:161-185

24. Sunitha V, Nirmala D, Srinivas C (2013) Extracellular enzymatic activity of endophytic fungal strains isolated from medicinal plants. World J Agric Sci 9(1):1-9

25. Katoch M, Salgotra A, Singh G (2014) Endophytic fungi found in association with Bacopa monnieri as potential producers of industrial enzymes and antimicrobial bioactive compounds. Braz Arch Biol Technol 57(5):714-722

26. Bric JM, Bostock RM, Silverstone SE (1991) Rapid in situ assay for indole acetic acid production by bacteria immobilized on nitrocellulose membrane. Appl Environ Microbiol 57:535-538

27. Pikovaskya RI (1948) Mobilization of phosphorus in soil in connection with vital activity of some microbial species. Mikrobiologiya 17:362-370

28. Schwyn B, Neilands JB (1987) Universal chemical assay for detection and determination of siderophores. Anal Biochem 160:47-56

29. Hormazabal E, Piontelli E (2009) Endophytic fungi from Chilean native gymnosperms: antimicrobial activity against human and phytopathogenic fungi. World J Microbiol Biotechnol 25:813-819

30. Dennis C, Wesbter J (1971) Antagonistic properties of speciesgroup of Trichoderma. III Hyphal interactions. Trans Brit Mycol Soc 57:363-369

31. Thakur JP, Haider R, Singh DK, Balagani SK, Vasudev PG, Luqman S, Kalra A, Saikia D, Negi AS (2015) Bioactive isochromenone isolated from Aspergillus fumigates, endophytic fungus from Bacopa monnieri. Microbiol Res 6:5800

32. Tamura K, Peterson D, Peterson N, Stecher G, Nei M, Kumar S (2011) MEGA5: molecular evolutionary genetics analysis using maximum likehood, evolutionary distance, and maximum parsimony methods. Mol Biol Evol 28(10):2731-2739

33. Jack IR, Okorosaye-Orubite K (2008) Phytochemical analysis and antimicrobial activity of the extract of leaves of fleabane (Conyza sumatrensis). J Appl Sci Environ Manage 12(4):63-65

34. Khanna VG, Kannabiran K (2008) Antimicrobial activity of saponins fractions of the leaves of Gymnema sylvestre and Eclip taprostrata. World J Microbiol Biotechnol 24:2737-2740

35 Wang G, Tang W, Bidigare RR (2005) Terpenoids as therapeutic drugs and pharmaceutical agents. In: Zhang L, Demain AL (eds) Natural products. Humana Press, Totowa, pp 197-227. https://doi. org/10.1007/978-1-59259-976-9_9

36. Jasim B, Daya PS, Sreelakshmi KS et al (2017) Bacopaside N1 biosynthetic potential of endophytic Aspergillus sp BmF 16 isolated from Bacopa monnieri. 3 Biotech 7:210

37. Kalyoncu F, Oskay M, SaÄŸlam H, ErdoÄ̈̈an TF, Tamer AU (2009) Antimicrobial and antioxidant activities of mycelia of 10 wild mushroom species. J Med Food 13:415-419

38. Lai HY, Yau YY, Kim KH (2010) Blechnum orientale Linn-a fern with potential as antioxidant, anticancer and antibacterial agent BMC Complement. Altern Med 10:15

39. Wu-Yang H, Yi-Zhong C, Xing J, Harold C, Sun M (2007) A potential antioxidant resource: endophytic fungi from medicinal plants. Eco Bot 61(1):14-30

40. Ramesha A, Srinivas C (2014) Antimicrobial activity and phytochemical analysis of crude extracts of endophytic fungi isolated 
from Plumeria acuminata L. and Plumeria obtusifolia L. Euro J Exp Bio 4:35-43

41. Bargah RK (2015) Preliminary test of phytochemical screening of crude ethanolic and aqueous extract of Moringa pterygosperma Gaertn. J Pharma Phytochem 4(1):7-9

42. Ayoola GA, Coker HAB, Adesegun SA, Adepoju-Bello AA, Obaweya K, Ezennia EC, Atangbayila TO (2008) Phytochemical screening and anti-oxidant activities of some selected medicinal plants used for malarial therapy in south western Nigeria. Trop J Pharma Res 7(3):1019-1021

43. Zhang YZ, Chen WF, Li M, Sui XH, Liu HC, Zhang XX, Chen WX (2012) Bacillus endoradicis sp. nov., an endophytic bacterium isolated from soybean root. Int J Syst Evol Microbiol 62(2):359-363

44. Selim K, El-Beih A, Abdel-Rahman T, El-Diwany A (2012) Biology of endophytic fungi. Curr Res Environ Appl Mycol 2(1):31-82

45. Pragathi D, Vijaya T, MouliK C, Anitha D (2013) Diversity of fungal endophytes and their bioactive metabolites from endemic plants of Tirumala hills-Seshachalam biosphere reserve. Afr J Biotechnol 12(27):4317-4323

46. Madhavi V, Lele SS (2009) Laccase properties, use. BioResources 4(4):1694-1717

47. Harrison RL, Bonning BC (2010) Proteases as insecticidal agents. Toxins 2:935-953

48. Murthy PS, Naidu M (2010) Protease production by Aspergillus oryzae in solid-state fermentation utilizing coffee by-products. World Appl Sci J 8:199-205

49. Chutmanop J, Chuichulcherm S, Chisti Y, Srinophakun P (2008) Protease production by Aspergillus oryzae in solid state fermentation using agro industrial substrates. J Chem Technol Biotechnol 83:1012-1018
50. Hassan SE (2017) Plant growth-promoting activities for bacterial and fungal endophytes isolated from medicinal plant of Teucrium polium L. J Adv Res 8:687-695

51. Dolatabad HK, Mohammad JN, Shier WT (2017) Evaluation of antifungal, phosphate solubilisation, and siderophore and chitinase release activities of endophytic fungi from Pistacia vera. Mycol Prog.https://doi.org/10.1007/s11557-017-1315-z

52 Tiwari S, Pandey R, Gross A (2021) Identification of rhizospheric microorganisms that manages root knot nematode and improve oil yield in sweet basil (Ocimum basilicum L.). Agronomy 11(3):570. https://doi.org/10.3390/agronomy11030570

53. Li E, Jiang L, Guo L, Zhang H, Che Y (2008) Pestalachlorides A-C, antifungal metabolites from the plant endophytic fungus Pestalotiopsis adusta. Bioorg Med Chem 16:7894-7899

54. Ramasamy K, Lim SM, Bakar AB, Ismail N, Ismail MS, Ali MF, Weber JFF, Cole ALJ (2010) Antimicrobial and cytotoxic activities of Malaysian endophytes. Phytothear Res 24(5):640-643

55. Elad Y (2000) Biological control of foliar pathogens by means of Trichodema harzianum and potential modes of action. Crop Prot 19:709-714

56. Sathiyabama M, Parthasarathy R (2018) Withanolide production by fungal endophyte isolated from Withania somnifera. Nat Prod Res 32(13):1573-1577

Publisher's Note Springer Nature remains neutral with regard to jurisdictional claims in published maps and institutional affiliations. 